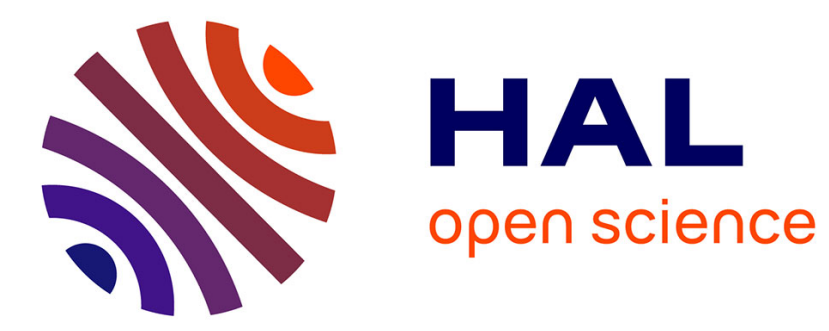

\title{
Expertises nomades au Sud. Eclairages sur la circulation des modèles urbains.
}

Éric Verdeil

\section{To cite this version:}

Éric Verdeil. Expertises nomades au Sud. Eclairages sur la circulation des modèles urbains.. Géocarrefour - Revue de géographie de Lyon, 2005, 80 (3), pp.165-169. 10.4000/geocarrefour.1143 . halshs-00094019

\section{HAL Id: halshs-00094019 \\ https://shs.hal.science/halshs-00094019}

Submitted on 9 Oct 2006

HAL is a multi-disciplinary open access archive for the deposit and dissemination of scientific research documents, whether they are published or not. The documents may come from teaching and research institutions in France or abroad, or from public or private research centers.
L'archive ouverte pluridisciplinaire HAL, est destinée au dépôt et à la diffusion de documents scientifiques de niveau recherche, publiés ou non, émanant des établissements d'enseignement et de recherche français ou étrangers, des laboratoires publics ou privés. 
Expertises nomades au sud. Eclairages sur la circulation des modèles urbains. Eric Verdeil, coordinateur du numéro, CNRS Environnement Ville Société, Lyon Géocarrefour, 2005, n80/3, pp.165-169

\section{Argument}

La réflexion sur la mondialisation a stimulé les recherches sur la diffusion des modèles d'aménagement et de gestion urbaine. Les échanges intra-européens, et euro-atlantiques, ont été souvent explorés (Rogers, 1998 ; Pinol et Walter, 2003). S. Ward, dans son ouvrage récent (2002, 395-408), en propose une typologie prenant en compte notamment les rapports de force géopolitiques. Une autre facette de cette diffusion des modèles concerne l'exportation des méthodes et modèles vers les pays colonisés (Goerg, Huetz de Lemps, 2003), et plus récemment, vers les pays en développement. Les experts et professionnels des pays occidentaux, les gestionnaires locaux ou les agents d'organisations internationales font partie des acteurs de cette circulation. Ils empruntent les canaux des coopérations bilatérales ou décentralisées (Saunier, 2002), ou agissent dans le cadre des organisations internationales (Osmont, 1995) de fréquents vecteurs des circulations.

L'accent a surtout porté sur les relations entre pays occidentaux ou sur les influences à partir de pays occidentaux. Envisager les circulation de modèles urbains dans une perspective plus large, et en réévaluer les principaux foyers ainsi que le sens et les mécanismes constitue l'objectif de ce dossier.

Un premier élargissement devrait consister en une prise en compte de la diversification des pays ou régions émetteurs de modèles. Ainsi, les interventions et les modèles japonais ou australiens tendent aujourd'hui à se développer puissamment en Asie. La coopération japonaise est active dans plusieurs pays du Moyen-Orient comme le Liban, la Syrie ou la Jordanie. Certains pays dits « émergents » deviennent aussi exportateurs de modèles urbains, via des firmes de BTP ou d'ingénierie. On peut citer la Corée du Sud (Fabre, 1999) ou le Liban (Verdeil, 2002, 438-495 et Ghosn 2005). La disparition de l'URSS tend à faire oublier que ses experts sont souvent intervenus non seulement eu Europe de l'Est mais dans nombre de pays de la galaxie socialiste comme l'Algérie, Cuba, le Vietnam, l'Egypte. Inversement, des techniciens originaires de ces pays ont été formés, non seulement à la révolution, mais à la pratique du développement. D’autres pays du bloc socialiste ont eux-mêmes été des modèles et des acteurs de la circulation des savoir-faire et des pratiques, comme en témoignent les parcours de professionnels syriens et jordaniens formés en RDA, en Pologne ou en Bulgarie (Longuenesse et Nasr, in Souami et Verdeil 2006 : 86, 90, 172). Par ailleurs, des expériences " alternatives », comme le budget participatif de Porto Alegre, les stratégies de transports collectifs de Curitiba (Macedo, 2004) ou de Singapour (Merlin, 1991) ou la diffusion, par l'intermédiaire de diverses agences internationales, des « Best Practices » dans le domaine de l'habitat, contribuent à faire reconnaître, au sud, des foyers d'innovation à partir desquelles sont diffusés des expériences et des modèles. Les mécanismes d’identification, de reconnaissance et enfin de mise en circulation de ces « modèles » méritent d'être identifiés. La diversification des lieux d'origine de ces modèles induit une seconde question : comment ces différentes influences se sont-elles mutuellement agencées, concurrencées, stimulées, superposées ou succédées?

Dans ce cadre, le regard doit se déplacer et ne plus se situer dans la logique de transfert « nord »-« sud », basée sur la métaphore de l'exportation, mais s'attacher également aux scènes locales qui jouent un rôle essentiel de filtrage, d'accomodation, de déformation et de recombinaison. Les contributions réunies par Joe Nasr et Mercedes Volait dans leur ouvrage 
Urbanism - Imported or Exported ? (2003), illustrent une telle perspective à partir d'études de cas situées dans des régions aussi diverses que l’Europe, la Méditerranée et le Moyen-Orient, l'Amérique latine, ou l'Extrême-Orient, et remontant jusqu'à 1850.

Une typologie des acteurs de la circulation identifie aussi des relais et intermédiaires, en différents endroits et instances, entre le lieu « émetteur » et le lieu « récepteur » : ces étapes de la circulation sont aussi des moments de reformulation et d'appropriation sélective des modèles. Bromley, (2004) et Harris (2004) le montrent chacun très bien à partir de l'étude de l'origine et du parcours complexe des idées prônées par John Turner, le " père » de self help, dont les écrits valorisant l'auto-construction dans les quartiers populaires sont communément considérés comme la source d'inspiration des organisations internationales et des nouvelles politiques d'habitat à partir des années soixante-dix. Jeune coopérant au Pérou, cet architecte britannique avait pu synthétiser les acquis de diverses expériences antagonistes. La réception favorable des idées de Turner ne s'explique que par le fait que de nombreux acteurs de l'aménagement urbain dans les pays pauvres y étaient déjà sensibilisés. Quant à la politique de sites and services (parcelles assainies) massivement soutenue par la Banque mondiale après 1973, dont le crédit est souvent porté à Turner, elle est bien davantage un compromis entre différentes expériences que la mise en œuvre pleine et entière des idées de ce dernier.

La figure du professionnel de l'urbanisme ou de la gestion urbaine représente l'un des axes d'analyse de ces scènes locales ou intermédiaires de la transmission de modèles. La circulation, de courte ou de longue durée, parfois dans le cadre de la migration, représente une dimension essentielle de la pratique de ces experts dans les pays du sud. Mobilité pour études ou pour formation continue, déplacement d'une aire géographique à l'autre, prenant parfois appui sur des diasporas constituées, carrière dans des organisations ou des firmes internationales, jeu sur les appartenances culturelles, les compétences linguistiques et les entre-deux géopolitiques, constituent autant de cadres et de modalités de circulation. Ils complexifient considérablement l'analyse classique des flux nord-sud et nuancent les schémas de domination économique et culturelle qu'on associe souvent à la mondialisation. La prise en compte d'une échelle micro, focalisant sur l'individu ou la firme et sur leurs motivations et leurs mobilités, présente l'avantage de mêler des déterminations socio-historiques globales et des enjeux souvent moins visibles, telles que des stratégies professionnelles, des dimensions sectorielles, des conjonctures locales. La carrière du consultant grec Constantinos Doxiadis (Bromley, 2003), figure illustre de la planification moderne, volant de Karachi à Bagdad, bénéficie à la fois de son inscription dans la mouvance états-unienne dans le contexte de guerre froide et de son profil d'intermédiaire culturel. L'étude de carrefours, comme certaines organisations internationales ou non-gouvernementales, est une autre manière de développer une telle perspective, comme un écran sur lequel sont projetés des flux de particules, dont la mise à plat à un moment donné permet de dresser une cartographie, de repérer des convergences ou au contraire, des processus d'évitement.

L’objectif de ce numéro de Géocarrefour est d'explorer certains de ces aspects méconnus des circulations de modèles urbains à travers le prisme des professionnels ou des experts. La présentation et la généalogie des modèles urbains ne se limitent pas à une histoire des idées et des techniques : celle-ci doit rendre palpables les dimensions concrètes des carrières et des trajectoires individuelles dont les interactions façonnent les discours et pratiques de l'aménagement, tout en les replaçant dans des conjonctures géopolitiques propres aux divers ensembles géographiques considérés.

Deux des contributions présentées proviennent des travaux menés dans le cadre d'une recherche intitulée « Cultures professionnelles de l’urbanisme au Moyen-Orient », sous les auspices de l’Institut français du Proche-Orient à Beyrouth, puis soutenue par le programme 
de recherche urbaine sur le développement (PRUD), dont une synthèse paraît aux éditions Anthropos-Economica (Souami, Verdeil, 2006). L’appel à contributions visait à élargir le champ d'enquête à d'autres espaces culturels, afin d'examiner d'autres acteurs, d'autres circulations et d'autres logiques. Cet élargissement enrichit les thématiques traitées et ouvre de nouvelles questions. Le dossier a ainsi une dimension exploratoire. Son ambition n'est pas tant de fournir une synthèse de connaissances que d'interroger un foisonnement d'expériences et d'échanges en matière de gestion urbaine et de susciter, en résonance, d'autres travaux.

\section{Des circulations sud-sud}

Les trois premiers textes apportent des éclairages sur des circulations d'idées, de pratiques et d'acteurs de l'aménagement urbain entre pays du sud, que nous avons plutôt l'habitude d'observer comme récepteurs ou importateurs de modèles urbains. Singapour, à la faveur de la transformation de son économie, devient une puissance économique régionale exportatrice. L'expertise acquise en matière de développement urbain sur son territoire alimente la dynamique d'investissements de firmes liées à l'Etat singapourien dans le domaine de l'immobilier dans plusieurs pays d'Asie orientale, de la Thaïlande et du Vietnam à la Chine. Xavier Guillot étudie la diffusion du condominium, un modèle d'habitat haut de gamme qui accompagne la mutation des modes de vie caractérisée par la montée d'une classe moyenne mondialisée, adoptant comme habitat le modèle du condominium. L'exportation du modèle du condominium par les entreprises singapouriennes est liée à une stratégie étatique de déploiement vers l'extérieur, dans le contexte d'une nouvelle organisation des flux économiques mondiaux et à la faveur de l'ascension de Singapour au statut de puissance économique régionale. Sa puissance financière se combine avec un modèle urbanistique et des structures opérationnelles élaborées pour l'aménagement du territoire singapourien. L'article montre que la stratégie d'exportation singapourienne s'appuie sur une élite technique locale anglophone auparavant en charge du développement urbain sur le territoire de la cité-Etat. Le deuxième article traite de l'action combinée des organisations internationales et nongouvernementales dans la résorption de l'habitat informel au Cambodge. Ce thème, comme le souligne l'exemple de Turner cité ci-dessus, fait l'objet de nombreux débats et expériences depuis un demi-siècle. Phnom Penh en reconstruction est un véritable carrefour de la coopération internationale. La conjonction des actions et des pressions de diverses ONG et des organisations internationales parvient à infléchir la politique gouvernementale d'éviction, même si dans les faits, face aux pressions des investisseurs et à la libéralisation économique encouragée par les mêmes bailleurs internationaux, de nombreuses contradictions apparaissent. L’originalité de l'étude vient de l'analyse très documentée des individus présents dans les ONG locales, qui appartiennent à divers réseaux d'ONG. Leur action est basée sur des échanges d'expériences entre communautés d'habitants de plusieurs pays asiatiques, réunies au sein d'un réseau progressivement élargi. Ces ONG produisent une contre-expertise qui est adoptée par les organisations internationales. Un double renversement se produit : ce ne sont plus des pratiques et des idées originaires des pays du nord mais d'autres, élaborées dans des pays du sud, qui sont prônées. La hiérarchie expert-habitant est aussi inversée. Les profils des militants promoteurs de ces nouvelles pratiques présentent une caractéristique essentielle : leur mobilité incessante entre sphères géographiques et culturelles, lors des études puis durant la pratique militante et professionnelle. Leur cooptation par des réseaux internationaux, y compris dans les organisations multilatérales, constitue un facteur de renouvellement de ces institutions dans leur composition nationale et, au moins partiellement, dans leur orientation idéologique. Il resterait à identifier les mécanismes de sélection de ces nouveaux experts et de leurs idées et à mieux comprendre les difficultés et les contradictions dans la mise en œuvre des modèles prônés sur le terrain, ce qu'amorce l'article de Valérie Clerc. 
Les deux exemples singapourien et cambodgien, à partir de perspectives très différentes et même inverses, illustrent la mise en place dans ces pays de politiques urbaines innovantes, fondées pour une part sur le réemploi d'outils ou de pratiques existants ailleurs, mais appropriés et adaptés par les acteurs locaux, qu'il s’agisse du gouvernement de la cité-Etat ou bien des leaders des communautés d'habitants et des responsables municipaux. La diffusion de ces pratiques n'emprunte pas les mêmes canaux et n’obéit pas aux mêmes finalités. La démarche des firmes para-étatiques singapouriennes s'inscrit dans une logique de conquête de marché. A l’inverse, l'écho donné aux expériences cambodgiennes repose sur des stratégies d'influence croisée de la part des ONG parties prenantes et des organisations internationales, visant à faire reconnaître une nouvelle culture de l'aménagement urbain. De ce point de vue, l'exemple cambodgien présente une analogie avec le prosélytisme de l'urbaniste palestinien Saba Shiber en faveur de nouvelles pratiques pour l'aménagement des villes arabes.

L'étude proposée par J. Nasr sur le parcours de cette personnalité s'inscrit néanmoins dans un double décalage : méthodologique, par l'usage de la biographie ; et chronologique, puisqu'on revient aux années soixante, âge de la planification triomphante et de son exportation aux quatre coins du monde au nom du développement. Shiber est véritablement un expert nomade, qui voyage entre la Palestine de son enfance, le Liban qui constitue, à l'âge adulte, son port d'attache, les Etats-Unis où il acquiert ses diplômes et son intérêt pour l'urbanisme, le Koweit qui devient son principal lieu d'exercice, et les multiples villes et pays où il est appelé pour conférences et consultations. A une époque où l'expertise en urbanisme, dans le Tiers monde, reste l'apanage de praticiens occidentaux, son parcours illustre à la fois la montée d'une expertise locale et la réappropriation, dans ce cas bien peu critique, des outils et de la vision dominante d'un aménagement urbain à visée universelle.

\section{Les milieux locaux de l'urbanisme face aux circulations d'expertises et de modèles urbains}

Les quatre autres articles adoptent une démarche différente où l'essentiel est moins dans les circulations des experts que dans le fait de scruter les modalités de réceptions d'expertises, de modèles et de pratiques d'aménagement, par des professionnels et des élus ou responsables politiques locaux. Cette posture permet de dégager deux idées centrales : le poids des milieux professionnels locaux dans l'intermédiation et le transit des idées, modèles et pratiques d’aménagement urbain en provenance de l'extérieur ; et le filtrage sélectif, voire le réassemblage de ces idées et pratiques (au sens du synthetic borrowing qu'évoque S.V. Ward, 2002, p.402) en fonction des contraintes, des compétitions politiques, des normes et des usages sociaux. Plutôt que de contribuer à une homogénéisation en fonction de modèles exportés depuis les pays occidentaux ou des organisations internationales, la circulation accrue des hommes et des idées suscite des appropriations et des adaptations spécifiques, ce qui conduit à reconsidérer la notion de modèle. On rejoint ici l'une des conclusions du dossier Gérer la ville : entre global et local à propos des services urbains (Dorier-Apprill, Jaglin 2002).

Les municipalités de Rosario en Argentine et de Montevideo en Uruguay sont étudiées par S. Velut et S. Robin. Dominées depuis la période de la démocratisation par des majorités de gauche, elles pourraient sembler des sites idéaux de réplication des modèles de Barcelone et de Porto Alegre. L'urbanisme barcelonais est devenu une icône glorifiée par les Jeux olympiques de 1992 et représente une vitrine des nouvelles pratiques politiques socialesdémocrates eu Europe. Ces pratiques ont obtenu en Amérique latine une forte résonance, grâce à la coopération espagnole et aux mécanismes d'aide de l’Union européenne. Porto Alegre est bien connu par les pratiques participatives valorisée par les milieux altermondialistes. Les parcours individuels d'urbanistes et intellectuels espagnols et ceux des révolutionnaires sud-américains se croisent à de nombreuses reprises depuis les années 
soixante-dix et contribuent à des connivences personnelles et à la diffusion de ces pratiques. Pourtant, les politiques de planification participative mises en œuvre dans les deux villes se distinguent par le poids prépondérant des facteurs locaux, luttes politiques et enjeux sociaux, dans leur mise en œuvre. Elles ne présentent plus, dès lors, que des analogies en grande partie rhétoriques avec les dits modèles.

J.M. Cusset s'intéresse aux nombreux bureaux d'études et firmes de services urbains installés au Vietnam, dans le contexte de libéralisation et d'investissements liés à une croissance urbaine soutenue. L'expertise vietnamienne est en mutation et traversée de tensions, entre des pratiques et des organisations héritées de la période socialiste et une recomposition liée à la libéralisation. Une percolation lente des idées des bureaux d'études étrangers n'exclut pas des relations tendues avec ses derniers : l'administration vietnamienne prend le temps de peser et de comparer les options que lui offrent des consultants, avant de les sélectionner en fonction de ses priorités.

A Abidjan, l'urbanisme reste marqué par des modèles formels occidentaux, symboles du miracle ivoirien. Ils sont réappropriés en tant qu'emblème d'une " certaine dignité urbaine ». Dans le contexte actuel de crise politique, la réactivation de ce modèle se traduit par une lutte contre l'informel et va de pair avec un discours nationaliste sur l'ivoirité. Jean-Fabien Steck examine les conditions de reproduction et de circulation de cette idéologie dans les lieux de formation et lieux d'exercice des ingénieurs et urbanistes ivoiriens, formés à l'étranger ou dont l'enseignement est modelé selon le modèle des écoles d'ingénieurs françaises. Il montre aussi leur diffusion dans les milieux politiques locaux.

Les pérégrinations de l'institut d'aménagement et d'urbanisme de la région Ile-de-France au Liban et en Egypte constituent ici pour T. Souami un prétexte pour observer les milieux professionnels locaux de ces deux pays à la fois en miroir l'un de l'autre, et au miroir de l'Autre, occidental, et des valeurs professionnelles qu'il véhicule. C'est l'occasion d'interroger les parcours et les références de quelques urbanistes du sud, leurs détours par le nord et leur convergence, avec les urbanistes français, autour du modèle professionnel de l'urbaniste.

Pour clore cette présentation, avançons quelques remarques transversales. La circulation accrue des experts et des expertises et la diversification de leurs lieux d'origine ne sont pas dissociables d'une recomposition des structures de domination économique et politique. L'expertise nomade n'est pas alors autre chose que l'expression privilégiée de ces rapports de force. On en trouve l'illustration dans la concurrence que se livrent les firmes et consultants dans le domaine des services urbains au Vietnam, appuyés par les instances de la coopération bilatérale. L'essor de Singapour et sa projection d'un modèle urbain en Asie orientale vont dans le même sens. La dimension linguistique et culturelle joue certainement comme un facilitateur de cette diffusion : les liens de l'Amérique latine avec l'Espagne ou l'influence française en Côte d'Ivoire montrent la permanence de ce facteur. Mais l'anglais s'affirme comme langue de contournement et de pénétration, non seulement dans le cas de Singapour, mais aussi pour un Arabe dans les pays arabes, ce dont témoigne l'exemple de Saba Shiber. Ces formes de domination n’imposent pas des « modèles » : localement, tout un travail de réappropriation est sans cesse à l'œuvre. Pour une part, ce travail est conditionné par les enjeux politiques et sociaux et les transformations dans la longue durée des sociétés locales. Pour le décrypter, les contributions de ce dossier montrent aussi l'intérêt d'un examen précis des scènes locales, et des différents acteurs qui les animent. La distinction entre experts, ceux qui proposent et savent, et politiques, ceux qui décident, est peu opératoire dès lors qu’on adopte une perspective biographique. Les parcours individuels montrent des changements de statuts entre différentes positions institutionnelles (étudiant, militant, expert, responsable élu, 
et cette énumération n’est pas nécessairement linéaire). Les différents parcours de militants et de responsables politiques cambodgiens étudiés par V. Clerc, tout comme ceux présentés par S. Velut et S. Robin à propos de l'Argentine et de l'Uruguay, en offrent des illustrations exemplaires. Ils permettent d'identifier des rencontres et des moments d'apprentissage d'idées et de pratiques, des périodes enfin de gauchissement et d'adaptation.

La labilité éventuelle des positions entre professionnels et politiques ne doit pas conduire à éclipser les enjeux proprement professionnels. Il faut au contraire les reconnaître. A travers la formation universitaire, et l'insertion dans un espace idéologique qui dépasse le local, le professionnel peut être un passeur d'idées. La compétition pour l'accès à la commande et la défense corporatiste expliquent aussi des préférences collectives pour certains " modèles », comme l'exemple de l'organisation des urbanistes ivoiriens le montre dans l'étude de J.F. Steck.

En conclusion, la circulation des expertises ne peut se comprendre uniquement à la lumière des rapports de force économiques et politiques globaux qui se dessinent dans la mondialisation actuelle. Elle suit des chemins éminemment complexes, dans le temps et dans l'espace, où les facteurs propres à la scène locale jouent un rôle majeur.

\section{Liste des références citées}

Bromley Ray , 2003, “Towards Global Human Settlements: Constantinos Doxiadis as Entrepreneur, Coalition-Builder and Visionary”, in Joe Nasr and Mercedes Volait (eds.), Urbanism : Imported or Exported. Foreign Plans and Native Aspirations, Chichester, Wiley, pp. 316-340

Bromley Ray, 2003, "Peru 1957-1977 : How Time and Place Influenced John Turner's Ideas on Housing Policy”, Habitat International 27 (2003), p.271-292.

Fabre Marie-Hélène, 1999, « L’urbaniste en Corée du Sud », Urbanisme, n³04, 1999, pp. 9192

Goerg Odile, Huetz de Lemps Xavier, 2003, « La ville européenne outre-mer », Livre 5, in JL Pinol, Histoire de l'Europe urbaine, vol. 2, De l'Ancien Régime à nos jours, Paris, Le Seuil, pp. 277-551.

Harris Richard, 2003, “A Double Irony: the Originality and Influence of John F.C. Turner”, Habitat International 27, p.245-269.

Dorier Apprill Elisabeth, Jaglin Sylvy (dirs.), 2002, « Gérer la ville. Entre global et local », Autrepart, $\mathrm{n}^{\circ} 21$.

Macedo Joseli, 2004, “City Profile: Curitiba”, Cities, vol.21, nº6, p. 537-549.

Merlin Pierre, 1991, Géographie et économie des transports, Paris, PUF.

Nasr Joe, Volait Mercedes (eds.), 2003, Urbanism : Imported or Exported. Foreign Plans and Native Aspirations, Chichester, Wiley, 372 p.

Osmont Annik, 1995, La banque mondiale et les villes : du développement à l'ajustement, Paris, Khartala, 309 p.

Pinol Jean-Luc et Walter François, 2003, « La ville contemporaine jusqu’à la Seconde Guerre mondiale », in Jean-Luc Pinol (ed.), Histoire de l'Europe urbaine, vol. 2, De l'Ancien Régime à nous jours, Paris, Ed. du Seuil, chap. 4 et 5, pp. 115-170

Rogers Daniel T., Atlantic Crossings. Social Politics in the Progressive Age, Cambridge, Harvard University Press, 1998, 672 p. ;

Saunier Pierre-Yves (ed.), 2002, 'Municipal Connections : Cooperation, Links and Transfers among European Cities in the 20th Century', Contemporary European History, Vol.11, No.4, November (Cambridge University Press).

Saunier PY, 2004, « Tel Mickey Mouse jouant au tennis . Charles S. Ascher et l'internationalisation de la public administration », Actes de la recherche en Sciences sociales, 151-152, pp. 49-57. 
Souami Taoufik, Verdeil Eric (dirs.), 2006, Concevoir et gérer les villes du sud. Milieux d'urbanistes, Paris, Anthropos-Economica, 230 p.

Verdeil Eric, 2002, Une ville et ses urbanistes : Beyrouth en reconstruction, these de géographie, Université de Paris 1, , 648 p.

Ward Stephen, 2002, Planning the Twentieth-Century City. The Advanced Capitalist World, Chichester, Wiley, 470 p. 